'Departamento de Psiquiatría y Salud Mental, Universidad de Concepción. Concepción, Chile. apsicóloga, Alumna de Doctorado de Salud Mental, Departamento de Psiquiatría y Salud Mental, Universidad de Concepción. Concepción, Chile.

Recibido el 12 de diciembre de 2019, aceptado el 15 de abril de 2020.

Correspondencia: Dr Benjamín Vicente Departamento de Psiquiatría y Salud Mental, Universidad de Concepción. Concepción, Chile. bvicent@udec.cl

\section{Plan nacional de salud mental. Reflexiones en torno a la implementación del modelo de psiquiatría comunitaria en Chile}

\author{
MARIELA GATICA-SAAVEDRA ${ }^{1, a}$, BENJAMÍN VICENTE ${ }^{1}$, \\ PATRICIA RUBÍ ${ }^{1}$
}

\section{Analysis of the new Chilean mental health care plan}

\begin{abstract}
During the sixties, the psychiatric care models evolved to a community care model, as a consequence of the asylum model failure. This new model assumes that psychosocial issues have a role, along with biological factors, in the development of mental diseases. In 2017 the National Mental health plan was created, based on this new model. It aimed to correct the flaws of the previous plan. We herein review this new plan, based on previous and international experiences. We also analyze the implementation of a community model of mental health care. The implementation of such a model without the support of the state and overlooking importance of mental health care, is extremely difficult.
\end{abstract}

(Rev Med Chile 2020; 148: 500-505)

Key words: Community Psychiatry; Mental Disorders; Mental Health; Public Health.

\section{I}

as primeras aproximaciones a la atención psiquiátrica se producen a través de instituciones totales, las cuales separan al enfermo mental de la sociedad, segregándolo, solo por el hecho de ser "diferente". En estos lugares la persona no recibía tratamiento, solo era aislado de la sociedad ${ }^{1}$.

En los años sesenta se comienza a producir una transformación en la comprensión de las enfermedades mentales lo cual desemboca en lo que más tarde se denomina una "reconversión de los sistemas de atención psiquiátrica", esto permite que se incorporen propuestas de distinto nivel a la intervención de salud ${ }^{2}$.

Entendiendo que la definición de la enfermedad mental es una construcción social, lo enfermo es variable según la cultura en la cual nos encontremos y la aproximación a esta va a depender de lo antes señalado ${ }^{3}$. En Chile tras la dictadura el servicio público de salud es casi inexistente, por lo tanto, se hace necesario escoger un modelo de salud mental que sea coherente con el retorno a la democracia ${ }^{4}$. En el año 1993 se presentan las primeras Políticas y Plan Nacional de Salud Mental el cual tiene principalmente como objetivo instalar el tema de salud mental en los servicios de salud y en los distintos niveles de atención. Este plan da pie en el año 2000 al nuevo Plan Nacional de Salud Mental y Psiquiatría el cual tiene como modelo de atención el modelo comunitario ${ }^{5}$.

El modelo comunitario aparece como un modelo respetuoso de los derechos humanos de los pacientes y en respuesta al fallo del modelo asilar ${ }^{5,6}$. Se asume que los factores biológicos son solo una parte del problema de salud mental ya que factores psicosociales tendrían influencia en el desarrollo y curso de la enfermedad mental ${ }^{4}$. Durante el periodo 2000 y 2017 se producen importantes avances 
en la implementación, creando dispositivos comunitarios, aumentando la dotación de personal en salud mental y desinstitucionalizando a los pacientes de los hospitales psiquiátricos, reduciendo los tiempos de hospitalización e insertando las camas psiquiátricas en hospitales generales. Este Modelo permite comprender la importancia del contexto en la recuperación de la persona, favorece su inclusión social y provee de las condiciones para el pleno ejercicio de sus derechos con el fin de mejorar su calidad de vida ${ }^{7}$.

El año 2017 se crea un nuevo Plan Nacional de Salud Mental el cual busca adaptarse a la reforma de salud que se ha ido implementando en Chile desde el año 2005 y la ratificación de la Convención Internacional de los Derechos de las Personas con Discapacidad. Este plan busca además corregir las brechas pendientes en cuanto a la implementación del modelo en el plan anterior.

Esta revisión es un análisis a partir de experiencias pasadas e internacionales del modelo comunitario y su implementación en Chile.

\section{El modelo comunitario en el nuevo plan nacional de salud mental}

El Ministerio de Salud entiende la Salud Mental como "la capacidad de las personas para interactuar entre sí y con el medio ambiente, de modo de promover el bienestar subjetivo, el desarrollo y uso óptimo de sus potencialidades psicológicas, cognitivas, afectivas y relacionales, el logro de sus metas individuales $y$ colectivas, en concordancia con la justicia y el bien común". Esta definición está adaptada del concepto desarrollado por el Ministerio de Salud de Canadá, y se centra en la persona, interactuando con su comunidad, funcionando en su pleno desarrollo y sobreponiéndose al concepto de medicina y psiquiatría, restringiendo la psiquiatría a la especialidad médica que se relaciona con la detección y recuperación de los trastornos o enfermedades mentales. Esta podría ser la razón por qué en esta versión del Plan de Salud Mental ya no se hace referencia a la Psiquiatría de manera explícita.

El nuevo plan sostiene que lo comunitario va más allá de un espacio físico, refiriéndose fundamentalmente a prácticas de intervención que reflejan las interacciones entre las diferentes redes sociales que constituyen la vida de un barrio. Por tanto, la atención de salud mental debería estar inmersa y participar de la realidad comunitaria, haciendo énfasis en la recuperación de la persona, la inclusión social y el ejercicio de derechos para así mejorar su calidad de vida. La persona tendrá más posibilidades de recuperarse si vive y es tratado en un ambiente parecido a su realidad ${ }^{8,9}$.

En relación a las fortalezas observadas en esta nueva propuesta se destaca la incorporación de los "determinantes sociales de la salud" los cuales se refieren a las circunstancias en que las personas nacen, crecen, viven, trabajan y envejecen, las cuales son el resultado de la distribución del dinero, el poder y los recursos ${ }^{7}$. Se entiende por tanto, que la mantención de la salud no tiene solo una base fisiológica sino que es multidimensional en donde aspectos sociales y económicos influirían en su restablecimiento. Esta mirada permite la incorporación de otros actores que originalmente no fueron parte de los equipos de salud como los trabajadores sociales, terapeutas ocupacionales, las organizaciones sociales y la comunidad. Lo anterior, complejiza las la intervención de salud mental al aportar con la mirada de otros profesionales los cuales poseen otros criterios de comprensión y aplican otras prácticas de atención sobre las personas ${ }^{2,7}$. Este modelo se enmarca en una mirada propuesta por la OMS dirigida al desarrollo de la persona en un ambiente igualitario desde el comienzo de la vida, considerando equidad de género, entornos urbanos adecuados, empleo y trabajo digno, protección social y atención sanitaria universal entre otros ${ }^{10}$. En la Estrategia Nacional de Salud 2011-2020, en uno de sus objetivos, se abordan específicamente seis factores determinantes de inequidades en salud: nivel socioeconómico, zona geográfica (lugar), pertenencia a pueblos indígenas, género, trabajo y condiciones de empleo, y pertenencia a otras poblaciones de interés (inmigrantes, personas privadas de libertad y personas en situación de calle) ${ }^{11}$.

Otra fortaleza es el "enfoque de curso de vida": aquí el acercamiento a la persona es en el contexto de un continuo vital inter relacional, donde hay una historia detrás que nos explica quién es la persona hoy (la suma de sus determinantes a través de la vida) y no solo está en manos de expertos que le dirán que hacer, sino que se va reescribiendo esta historia considerando lo que ha sido. Este enfoque consideraría por tanto, poner atención en los aspectos que podrían afectar al desarrollo de la enfermedad y no solo al curso de esta. 
Otro aspecto destacable es el modelo de dos continuos, en el cual el nivel de salud mental se representa en un eje vertical que va desde un "bajo nivel de salud mental" hasta una "salud mental óptima", y un eje horizontal que abarca desde la ausencia de diagnóstico y/o sintomatología de enfermedad mental, hasta la presencia de una enfermedad mental grave y compleja. El modelo por tanto, permite entender que a través del curso de vida de una persona esta pueda vivir con un diagnóstico de una enfermedad mental compleja, pero con un buen nivel de salud mental. Por otra parte, considera la necesidad de intervención en personas que no presentan un diagnóstico de enfermedad mental pero que vivan a través de su vida con un bajo nivel de salud mental.mSi se mantiene la política de cumplimiento de número atenciones de salud en patologías previamente determinadas para alcanzar metas sanitarias, las personas que estén en el cuadrante antes señalado, nunca recibirán atención.

Se observa por tanto, que el modelo comunitario presenta una ideología relacionada principalmente con el pleno desarrollo de la persona, en su comunidad, en el ejercicio de sus derechos. Se aleja del concepto de enfermedad tal como lo concebía el modelo biomédico, dejando de ser la salud mental por tanto, un ámbito exclusivo de los médicos, entendiéndola desde el contexto en el que se encuentra la persona y como, siendo parte de este y conociéndolo, la intervención podría ser más efectiva. Incorpora además la interdisciplinariedad, inter institucionalidad, inter sectorialidad y la participación social como aspectos importantes en la intervención y recuperación de la persona ${ }^{2,7}$.

Para dar forma a esta propuesta, el estado desde el año 2000 ha realizado importantes inversiones en infraestructura, gestión y recursos humanos. Sin embargo, tras la evaluación realizada en el año 2014, queda en evidencia la existencia de importantes brechas que no permitirían la implementación completa del modelo y las cuales buscarían ser corregidas en el último Plan de Salud Mental ${ }^{7,9}$.

Con respecto a la financiación del programa, casi todas las líneas de acción implican una expansión en la cobertura de salud a nivel prevención, promoción e intervención, sin embargo, el presupuesto se reduce porcentualmente de manera progresiva cada año. Esto puede deberse a que no existe claridad con respecto al gasto relacionado con: promoción y prevención, salud mental en el
Programa de Prestaciones Institucionales, intervenciones comunitarias, capacitación, gasto por grupos poblacionales, por patología, prestadores privados con y sin fines de lucro y gestión, sumado a lo anterior, el Estado financia acciones de salud mental que son ejecutadas por diversos entes públicos, sin a veces contar con la supervisión técnica de Salud ${ }^{7}$. Frente a este antecedente necesario para solicitar un financiamiento adecuado, es difícil exigir al nivel central mejoras en cuanto a presupuesto. El nuevo plan busca instalar sistemas de información sobre recursos en salud mental y fortalecer e identificar mecanismos de financiación de manera de poder llegar a $6 \%$ del presupuesto de salud.

Por otra parte, no existen estudios recientes de prevalencia de patología mental y al no tener mediciones del impacto de las políticas de salud mental implementadas, no es posible tener un panorama más completo sobre la evolución de la salud mental en el país y la efectividad de los recursos invertidos ${ }^{12}$.

El porcentaje del presupuesto de salud en el sistema público que se destinó a salud mental en el 2015 fue de $2,13 \%$, cifra que es aproximadamente la misma que se destinaba en el año 2004 (2,14\%) y alcanzando el monto más alto el año 2008 que llegó a $3,1 \% \%^{7,9}$. No se encuentran disponibles las cifras en relación al monto total. Lo anterior, deja en evidencia la mínima priorización que entrega el estado a la salud mental considerando que países como Estados Unidos y Canadá destinan 6,2\% y $7,2 \%$ respectivamente ${ }^{13}$.

La experiencia española en relación a salud mental muestra que el estancamiento de la calidad asistencial se debe a un crecimiento insuficiente de la financiación y del desarrollo de recursos específicos para atender la salud mental. Lo anterior, ha repercutido en el estancamiento de los centros de salud mental (CSM) comunitarios, no pudiendo por ejemplo, reducir las necesidades de hospitalización (14). De no mejorarse estos aspectos, probablemente estas dificultades se observen en el corto plazo en el modelo chileno.

La Organización Mundial de la Salud ubica a Chile entre los países con mayor carga de morbilidad por enfermedades psiquiátricas $(23,2 \%)$ en el mundo ${ }^{13}$. La recomendación de la OMS es invertir en salud mental ya que al hacerlo, no solo repercute positivamente en la persona reduciendo la discapacidad, el estigma y la muerte prematura, 
si no que ayuda además, a aumentar el capital social, a reducir la pobreza y a promover el desarrollo del país ${ }^{15}$.

En relación a la línea provisión de servicios, el nivel primario de atención ha alcanzado un amplio desarrollo en cuanto al crecimiento de recursos humanos e instalación de programas de intervención ${ }^{7}$. Se encuentra además, territorialmente inserto en la comunidad, sin embargo, las acciones de prevención y promoción en salud pareciera no ser lo suficientemente efectivas, lo cual se observa en la alta prevalencia de trastornos mentales en nuestro país ${ }^{16}$. Otra crítica tiene relación con las atenciones centradas en el cumplimiento de metas sanitarias provocando que el programa de salud mental se aleje del contacto real con la comunidad y pierda la oportunidad de diagnosticar y tratar de forma precoz los problemas de que en ella se presentan. El nuevo plan no prioriza áreas de atención en oposición al plan anterior que definió seis áreas prioritarias, sin embargo, solo cuatro (depresión, trastorno bipolar, esquizofrenia y abuso/dependencia de alcohol y drogas) han recibido mayor apoyo político y financiamiento suficientes a través de la incorporación a AUGE/ GES, sumándose recientemente la enfermedad de Alzheimer ${ }^{12,17}$.

Con respecto a la atención en el nivel secundario, ha habido importantes avances en cuanto a cobertura de atención ambulatoria. Se propone como centro especializado para este nivel de atención los Centros Comunitarios de Salud Mental (COSAM), como forma de acercar al equipo de Salud Mental a la comunidad, priorizándolo por sobre los equipos ambulatorios que funcionan en los hospitales generales, proponiendo incluso la desaparición de estos y su conversión a COSAM.

El hecho de que un Centro de Salud Mental funcione inserto en la comunidad puede tener aspectos a favor y otros en contra. A favor se encuentra el hecho de que estar en la comunidad, por lo tanto debería conocer sus características y bridar una atención coherente con las fortalezas y debilidades que esta presenta. Si bien el COSAM está planteado con el objetivo de disminuir la estigmatización del paciente de salud mental ${ }^{14}$, en nuestro país esto no siempre ocurre ya que el centro es identificado como tal en la comunidad, reproduciendo de algún modo "moderno" el modelo asilar, apartando a la persona del resto de las atenciones de salud. Otra dificultad tiene relación con la laxitud de las líneas de atención las cuales son: programa de adicciones, programa adulto y programa infanto-juvenil, presentando entre un centro y otro una amplia variabilidad en cuanto a la composición de los equipos y prestaciones. Por último, se dificulta el acceso a la atención en salud física al sacar la atención en salud mental de los centros de salud hospitalarios, confinándola a un área, y obviando por tanto el principio de atención integral en salud restringiéndolo solo al ámbito de la salud mental.

Con respecto a la atención terciaria, se ha logrado la disminución de los hospitales psiquiátricos y se han ampliado las camas psiquiátricas en hospitales generales. Aún cuando se busca evitar la institucionalización, en el momento en el cual se presenta el Plan 2017-2020 no existían guías o normas en relación a qué intervenciones terapéuticas debiera recibir el paciente hospitalizado $^{7}$. Desde el 2017, el Ministerio de Salud presentan las "Orientaciones para la Planificación y Programación en Red", de las cuales el anexo 18 "Atención cerrada de especialidad en Salud Mental” estaría dirigidos a suplir este déficit ${ }^{18}$. Finalmente, aún cuando existen, los dispositivos comunitarios destinados a recibir al paciente al alta y apoyarlo en la rehabilitación no serían suficientes ni están equipados de manera adecuada, interfiriendo por tanto en la continuidad del cuidado. El nuevo plan plantea trabajar para reducir estas brechas.

Con respecto a la calidad de la atención, en el último Plan de Salud Mental se señala la importante brecha que existe en este aspecto y propone formas de corregirlo. Las evaluaciones del modelo lo han hecho considerando las brechas de implementación pero sin considerar la calidad de las prestaciones entregadas ${ }^{7}$. Implementar un modelo de atención de alto estándar sin considerar la evaluación de la calidad de las atenciones prestadas es en estos momentos una falencia importante. La OMS plantea que enfocarse en la calidad ayuda a asegurar la optimización de los recursos, los cuales son generalmente escasos. La calidad asegura efectividad y eficiencia y además, cimientos sólidos para el futuro desarrollo del servicio ${ }^{19}$.

Finalmente, con respecto a la intersectorialidad, entendiendo la ideología a la base del modelo, es imposible que este sea eficiente y efectivo sin la participación de otros sectores de la comunidad y del mismo gobierno. 


\section{Reflexión crítica y propuestas}

Es importante visualizar la mirada filosófica desde la cual se posiciona el Ministerio de Salud para la propuesta de atención en salud mental. La construcción de la enfermedad no es desde el estado de enfermedad, si no desde un continuo restablecimiento del equilibrio y hace al estado responsable de esto y no solo a la persona. Incorpora los "determinantes sociales de la salud" como responsable de la mantención de este equilibrio, esto implica mirar más allá de la satisfacción de necesidades básicas y visualizar aspectos sociales y económicos los cuales dan contexto y determinan la presencia y evolución de una enfermedad. Muchas patologías psiquiátricas están afectadas por la desigualdad y la creación de nuevas necesidades que se proyectan en artefactos que no están al alcance de todos poseer y que en consecuencia generan malestar. El estado por tanto si quiere ser parte del restablecimiento del equilibrio, debería dirigir parte de sus esfuerzos en reducir brechas sociales y económicas.

Por otra parte, Chile posee un modelo económico neoliberal pero para que el modelo comunitario pueda ser implementado, es necesario un sistema de salud y de servicios sociales públicos, que garanticen prestaciones universales y equitativas, donde funcione la complementariedad y la colaboración y no la competencia propia del libre mercado ${ }^{20}$.

Impresiona en la implementación del modelo y en la revisión de este una falta de autocritica. Las críticas están restringidas a fallos en la implementación estructural (recursos humanos, capacitación, infraestructura) pero se diluye la ideología que lo sustenta. Llama la atención la queja constante de la falta de presupuesto para sostener el modelo, sin embargo, no existen datos concretos que permitan argumentar el aumento del aporte fiscal. La falta de financiamiento visibiliza el estigma y discriminación hacia las personas que viven con trastornos mentales desde el sistema de salud, haciendo patente las diferencias en el acceso a tratamiento de enfermedades físicas versus las mentales ${ }^{12}$. Surge por tanto las preguntas: idesde dónde estamos mirando la salud mental en Chile?; ¿es adecuado implementar un modelo comunitario de salud mental en un país con importantes brechas de desigualdad?; ¿ es posible implementar el modelo comunitario sin hacer parte al resto de los actores del estado?; ¿estaba preparado el país para la implementación del modelo?.

$\mathrm{Al}$ enfrentarse a la realidad de la atención en el Sistema Público de salud impresiona que gran parte de las propuestas del ministerio a través del Plan de Salud Mental son utópicas, una especie de manifiesto de trabajo, sin que existan los recursos económicos suficientes para su implementación. Sumado a lo anterior, para intervenir los "determinantes sociales de la salud", se debería considerar el trabajo intersectorial, es decir que el resto de los ministerios conciba la salud mental tal como lo hace el Ministerio de Salud, para poder así realizar un trabajo conjunto. Lo antes señalado se complementa también con el concepto de "salud en todas las políticas" la cual plantea se debiera adoptar un enfoque intersectorial de las políticas públicas teniendo en cuenta las repercusiones sanitarias de estas, promoviendo la sinergia y evitando efectos perjudiciales ${ }^{21}$. Si el foco de acción de la salud fuera sobre los "determinantes sociales de la salud", esto se traduciría en la reducción de las inequidades en salud a través del acceso oportuno, equitativo y de calidad para la población ${ }^{22,23}$ situación que a partir de lo señalado previamente, en Chile no ocurre. Pareciera que no existe conciencia de que el capital de un país se basa en las personas que lo constituyen y que "no hay salud sin salud mental".

\section{Referencias}

1. Foucalt M, Historia de la locura en la época clásica. México DF, México: Fondo Nacional de la Cultura Económica; 1970.

2. Galende E, De un horizonte incierto: Psicoanálisis y salud mental en la sociedad actual. Buenos Aires, Argentina: Paidós;1997.

3. Vicente B. La reforma psiquiátrica italiana. Rev de Psiquiatría 1991;8: 936-39.

4. Minoletti A, Zaccaria A. Plan nacional de salud mental en Chile: 10 años de experiencia. Rev Panam Salud Publica. 2005;18(4-5):346-58.

5. Ministerio de Salud (Chile). Plan nacional de salud mental y psiquiatría. Santiago; 2000.

6. Vicente B, Rioseco P, Vielma M, Pihan R, Muñoz M. Análisis ético de los modelos de atención de los pacientes crónicos. Rev de Psiquiatría 1989; 6 (2): 121-25.

7. Ministerio de Salud (Chile). Plan Nacional de Salud Mental 2017-2025. Santiago; 2017.

8. Torres R. Experiencias de psiquiatría comunitaria en 
Chile. Santiago: Cursos de educación continua Gestión en psiquiatría y salud mental; 2001. Disponible en http://www.bvsde.paho.org/texcom/cd050644/torres.pdf

9. Ministerio de Salud (Chile). Sistema de Salud Mental en Chile. Segundo informe WHO AIMS. Santiago; 2014. Disponible en http://www.who.int/mental_health/ who_aims_country_reports/who_aims_report_chile.pdf

10. Organización Mundial de la Salud (OMS). Subsanar las desigualdades en una generación: alcanzar la equidad sanitaria actuando sobre los determinantes sociales de la salud: informe final de la Comisión Sobre Determinantes Sociales de la Salud. Buenos Aires: Ediciones Journal; 2009.

11. Ministerio de Salud (Chile). Estrategia Nacional de Salud. Para el cumplimiento de los objetivos sanitarios de la década 2011-2020. Santiago; 2011.

12. Errázuriz P, Valdés C, Vöhringer PA, Calvo E. Financiamiento de la salud mental en Chile: una deuda pendiente. Rev Med Chil. 2015;143:1179-86.

13. Vicente B, Saldivia S, Pihán R. Prevalencias y brechas hoy: salud mental mañana. Acta Bioeth. 2016;22(1):5161.

14. Knapp M, Mcdaid D, Mossialos E, Thornicroft G. Salud mental en Europa: políticas y práctica. Líneas futuras en salud mental. Barcelona: Open University Press en nombre del Observatorio Europeo de Políticas y Sistemas Sanitarios; 2007.

15. Organización Mundial de la Salud. Invertir en salud mental. Ginebra; 2004. Disponible en https://www.who. int/mental_health/advocacy/en/spanish_final.pdf

16. Saldivia S. Prevalencia y variables asociadas a trastornos mentales comunes en centros de atención primaria de la provincia de Concepción [Internet]. Universidad de Chile; 2016. Disponible en: http://bibliodigital.saludpublica.uchile.cl:8080/dspace/handle/123456789/487

17. Minoletti A, Rojas G, Horvitz-Lennon M. Salud mental en atención primaria en Chile: aprendizajes para Latinoamérica. Cad Saúde Coletiva. 2012;20(4):440-7.

18. Ministerio de Salud (Chile), Subsecretaría de Redes Asistenciales. Orientaciones para la planificación y programación en red 2020. Santiago:2019. Disponible en https://www.minsal.cl/wp-content/ uploads/2019/09/2019.09.09_ORIENTACIONES-PARA-LA-PLANIFICACION-EN-RED-2020_v3.pdf

19. Organización Mundial de la Salud. Mejora de la calidad de la salud mental. Ginebra: Editores Médicos S.A.; 2003.

20. Desviat M. Panorama internacional de la reforma psiquiátrica. Cien Saude Colet. 2011;16(12):4615-21.

21. Organización Mundial de la Salud. Todo lo que necesitas saber sobre la salud en todas las políticas. Disponible en https://www.who.int/social_determinants/publications/health-policies-manual/key-messages-es.pdf?ua $=1$

22. Flacso Chile. La Construcción de la intersectorialidad-salud en todas las políticas desde la perspectiva de equidad y Determinantes Sociales de la Salud. Disponible en https://www.minsal.cl/sites/default/files/ La_construccion_intersectorialidad_salud.pdf

23. Ministerio de Salud (Chile), Subsecretaría de Redes Asistenciales. Promoción de Salud. Disponible en https://www.minsal.cl/wp-content/uploads/2016/09/1_ PROMOCION-DE-SALUD.pdf 